\title{
Correction to: A candidate gene study of intermediate histopathological phenotypes in HIV-associated neurocognitive disorders
}

\author{
Andrew J. Levine ${ }^{1}$ - Virawudh Soontornniyomkij ${ }^{2}$ - Eliezer Masliah ${ }^{3}$ • Janet S. Sinsheimer ${ }^{4,5} \cdot$ Sarah S. Ji ${ }^{5}$. \\ Steve Horvath ${ }^{5,6}$. Elyse J. Singer ${ }^{1}$ - Asha Kallianpur ${ }^{7}$. David J. Moore ${ }^{2}$
}

Published online: 6 July 2020

(C) Journal of NeuroVirology, Inc. 2020

\section{Correction to: Journal of NeuroVirology https://doi.org/10.1007/s13365-020-00846-Z}

Due to a production error data in Table 1 were not presented correctly. The corrected table is shown below.

The online version of the original article can be found at https://doi.org/ 10.1007/s13365-020-00846-Z

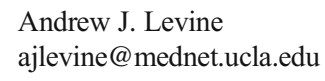

1 Department of Neurology, David Geffen School of Medicine at the University of California, Los Angeles, CA, USA

2 Department of Psychiatry, University of California San Diego School of Medicine, San Diego, CA, USA

3 Departments of Neurosciences and Pathology, University of California San Diego School of Medicine, San Diego, CA, USA
Departments of Human Genetics and Computational Biology, David Geffen School of Medicine at the University of California, Los Angeles, CA, USA

5 Department of Biostatistics, UCLA Fielding School of Public Health, Los Angeles, CA, USA

6 Department of Human Genetics, David Geffen School of Medicine at the University of California, Los Angeles, CA, USA

7 Department of Molecular Medicine, Genomic Medicine, Medicine, \& Pediatrics, Cleveland Clinic/Lerner Research Institute, Cleveland Clinic Lerner College of Medicine of Case Western Reserve University, Cleveland, OH, USA 
Age at death

Length of HIV infection

CD4+ T cell count

Median plasma HIV-1 RNA viral load

CPE

Global clinical rating

Detectable ( $>50$ copies $/ \mathrm{mL}$ ) plasma HIV-1 RNA viral load

Male

Race/ethnicity

Caucasian

African American

Hispanic

Asian/Native Alaskan

Major depression

Current

Past

Substance use disorder

Current

Past

Alcohol use disorder

Current

Past

HIV encephalitis

HAND

ANI

MND

HAD
47.4 years $^{\mathrm{a}}(\mathrm{SD}=9.2)$

12 years $(\mathrm{SD}=6.4)$

$122(\mathrm{SD}=168)$

15,406 copies $/ \mathrm{mL}$

$8.96(\mathrm{SD}=4.08)$

$5.31(\mathrm{SD}=1.87)$

Percent ${ }^{\mathrm{b}}$ of sample $(n)$

$84 \%$ (30)

$81.4 \%(162)$

$50.8 \%(100)$

$24.9 \%$ (49)

$22.3 \%$ (44)

$2 \%(4)$

$23.4 \%(32)$

$16.8 \%(23)$

$20.3 \%(31)$

$29.4 \%(45)$

$9 \%$ (14)

$26 \%(40)$

$9.2 \%(16)$

$74.2 \%$ (135)

$14.2 \%(26)$

$31 \%(56)$

$29 \%(53)$

$S D$ standard deviation, $C P E$ central nervous system penetration or effectiveness, ANI asymptomatic

Publisher's note Springer Nature remains neutral with regard to jurisdictional claims in published maps and institutional affiliations. 